Scientific Paper

\title{
A supine cranio-spinal irradiation technique using moving field junctions
}

\author{
Karthick Raj MANI ${ }^{1,2}$, Shantanu SAPRU ${ }^{3}, \mathrm{KJ} \mathrm{J} \mathrm{MARIA} \mathrm{DAS}^{2, a}$, Ayan BASU ${ }^{4}$ \\ ${ }^{I}$ Research \& Development Centre, Bharathiar University, Coimbatore - 641 046, Tamilnadu, India \\ ${ }^{2}$ Department of Radiotherapy, Sanjay Gandhi Postgraduate Institute of Medical Sciences, Rai Bareily Road, \\ Lucknow - 226014, Uttar Pradesh, India \\ ${ }^{3}$ Department of Radiation Oncology, Dr. Ram Manohar Lohia Institute of Medical Sciences, Gomtinagar, \\ Lucknow, Uttra Pradesh, India \\ ${ }^{4}$ Department of Radiation Oncology, The Mission Hospital, Durgapur - 713212, West Bengal, India \\ ${ }^{a}$ E-mail address: kjmariadas@hotmail.com
}

(received 30 May 2016; revised 8 September 2016; accepted 17 October 2016)

\begin{abstract}
Aim: To demonstrate a simple technique of cranio-spinal irradiation (CSI) in supine position using inter fraction moving field junctions to feather out any potential hot and cold spots.

Materials and Methods: Fifteen patients diagnosed with medulloblastoma were treated during the period February 2011 to June 2015 were included in this study. Out of fifteen patients in the study nine were male and 6 were female with a median age of 13.4 years (range 5-27 years). All the patients were positioned supine on CT simulation, immobilized using thermoplastic mask and aligned using room based laser system. Two parallel opposed lateral fields for the whole brain using an asymmetrical jaw with isocenter at $\mathrm{C} 2$ vertebral body. A posterior field also placed to cover the cervical and dorsal field using the same isocenter at C2. The second isocenter was placed at lumbar vertebral region to cover the remaining dorsal, lumbar and sacral region using an inter-fraction moving junction. Field-in-field and enhanced dynamic wedge used to homogeneous dose distribution when required.

Results and Discussion: In this study, we found that only two patients failed in the primary site, no radiation myelitis or recurrences in the filed junctions were reported in these fifteen patients with a median follow-up of 36.4 months. The automated sequence of treatment plans with moving junctions in the comfortable supine position negating the need for manual junction matching or junction shifts avoiding potential treatment errors and also facilitating delivery of anesthesia where necessary.
\end{abstract}

Key words: CSI; medulloblastoma; supine.

\section{Introduction}

Medulloblastoma, comprising $20 \%$ of childhood brain tumors, is conventionally treated with maximal safe resection followed by irradiation to the entire cranio-spinal axis along with boost to the posterior fossa and sites of radiologically documented gross deposits or seeding in the lepto-meningeal region with sequential chemotherapy used in select high risk cases [4]. Cranio-spinal irradiation (CSI) is an intrinsically challenging radiotherapy technique fraught with difficulties arising from the need to ensure adequate and complete coverage of the brain, spinal cord and entire thecal sac which in turn has an important bearing in the eventual outcome of the treatment. Treatment delivery in the uncomfortable prone position is difficult in children shortly after surgery and anaesthetization in the said position is a difficult prospect when required. Improper treatment due to inadequate immobilization resulting from patient discomfort may result in either under-dosage to treatment volumes or over-dosage to the critical spinal cord, resulting in either recurrences or radiation myelopathy [3]. Supine position is preferred for radiotherapy treatment of most anatomical sites due to patient comfort and stability ensuring reproducibility of daily patient set-up and less dosimetric variation. The prone position is usually chosen due to the irradiated area of interest being the posteriorly placed vertebral canal region and because in the era before the advent of CT scanners and TPS-based planning, planning was done by manual landmark assessment which could be done only in the prone position. Other critical issues include junction between cranial and spinal fields, adequate coverage of cribriform plate and temporal fossa which present inherent difficulties in the erstwhile practiced $2 \mathrm{D}$ techniques, resulting in progressive adaptation of $3 \mathrm{D}$ conformal treatment in this clinical scenario $[1,5]$.

Several authors like Parker et al [2] have tried to adopt the CSI for the supine position to circumvent the above problems. However, theirs was a junction matching technique, rather than a moving junction technique. We simulated the Parker 
technique in which the collimator was rotated by $11.2^{\circ}$ in the cranial fields, to match the spinal field divergence. We observed that a mere $2 \mathrm{~mm}$ mismatch in the longitudinal direction resulted in considerable dosimetric variability that ranged from a $45 \%$ hot-spot (in case of an overlap) to a $30 \%$ cold-spot (in case of a gap) between the junctions (Figure 1 and 2). This proposed junction match technique requires a submillimeter mechanical accuracy of the machine and a very rigid immobilization system to assure accurate and safe dose delivery. The moving junction technique intuitively seems to have less dosimetric variability and also has the added advantage of feathering out the dosimetric effects of any hot or cold spots at the field junctions. Yom et al [6] also discussed the cranio-spinal irradiation using field-in-field technique homogenization with daily intra-fractional modulation of the field junctions, to minimize the possibility of spinal cord overdose. South et al [7] discussed about the advantage of the supine position for cranio-spinal irradiation include patient comfort, easier access to maintain an airway for anesthesia and reduced variability of the head tilt in the face mask.

To address the above problems, we attempted to develop a practical treatment planning and delivery approach for the cranio-spinal irradiation in the supine position using the reliable and safe moving junction technique.

\section{Materials and Methods}

\section{Immobilization and Data Acquisition}

Fifteen patients diagnosed with medulloblastoma were treated during the period February 2011 to June 2015 using this moving field junction technique. Patients were placed supine on carbon fiber overlay couch top of a CT-simulator and aligned using room lasers. A ' $U$ ' type thermoplastic mask was used to immobilize the head on an appropriate neck rest ensuring maximal extension of neck to make the spine straight. Three fiducial markers were placed on the mask to define patient co-ordinates in the treatment planning system. Tattoos were marked on the sternum and pubic symphysis using the

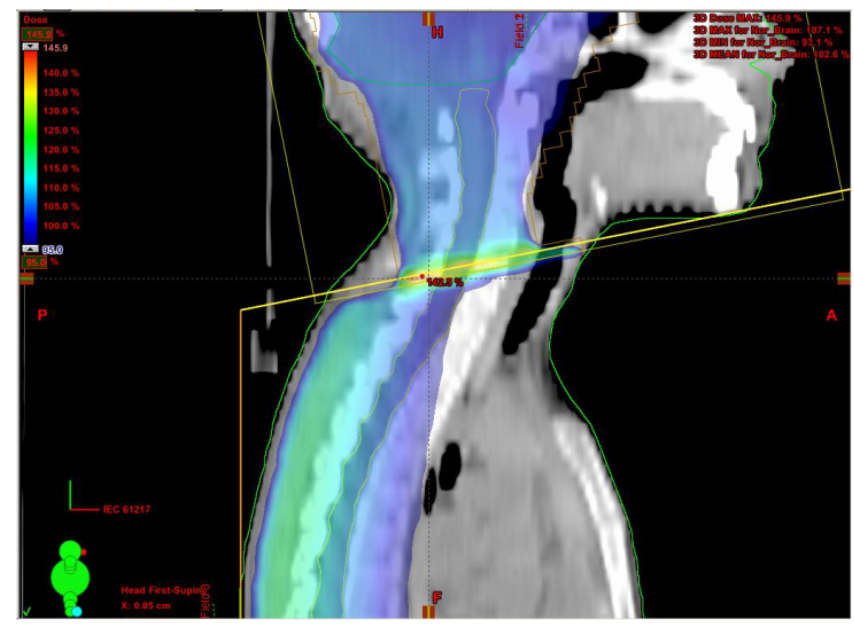

Figure 1. Simulation of matching junction technique demonstrating $2 \mathrm{~mm}$ overlap leads to $45 \%$ overdose at the junction. sagittal room laser for reproducible patient positioning. Patients were then scanned in the CT-simulator, from the vertex till ischial tuberosities, using $3 \mathrm{~mm}$ slice thickness, with a $430 \mathrm{~mm}$ FOV with a pitch of 1 .

The CT data was transferred to the Eclipse treatment planning system (TPS) (M/S Varian Medical Systems Inc, Palo Alto, CA) and a 3D dataset was generated. Where possible, MRI scan of the brain region was used for fusion with planning CT to aid delineation of target volumes, using following parameters: scan area from vertex to $\mathrm{C} 2$ area; slice thickness of $2 \mathrm{~mm}$; FOV of $260 \mathrm{~mm}$; with no inter-slice spacing. Gadolinium-enhanced T1 contrast and T2-FLAIR sequences were imported into the TPS. A separate screening MRI of the entire spine was acquired to rule out disseminated disease.

\section{Target Delineation}

The MRI data was fused with the CT-RTP data using the coregistration module of the Eclipse TPS (Rigid Registration Algorithm 8.6.15). The entire brain (CTV_Brain) was delineated using auto-contouring tools and modified manually if necessary. The entire spinal canal was identified as the CTV for treatment and was delineated as the CTV_Spine. A separate boost volume (CTV_Boost) was drawn as visualized on the post-operative MRI or in cases where this was not feasible by using the pre-operative MRI imaging available. A uniform $0.5 \mathrm{~cm}$ PTV margin was then generated for the CTV_Brain (PTV_Brain), and a $1 \mathrm{~cm}$ margin for the CTV_Spine (PTV_Spine) (except in the anterior and posterior directions, where it was restricted to $0.5 \mathrm{~cm})$. A separate $0.5 \mathrm{~cm}$ margin is generated for the CTV_Boost (PTV_Boost). We ensured that the CTV_Brain does not overlap with the CTV_Spine and also that the PTV_Brain does not overlap with the PTV_Spine by delimiting the latter where the first ends cranio-caudally. If required the PTV_Brain was cropped $0.5 \mathrm{~cm}$ from the 'Body' contour.

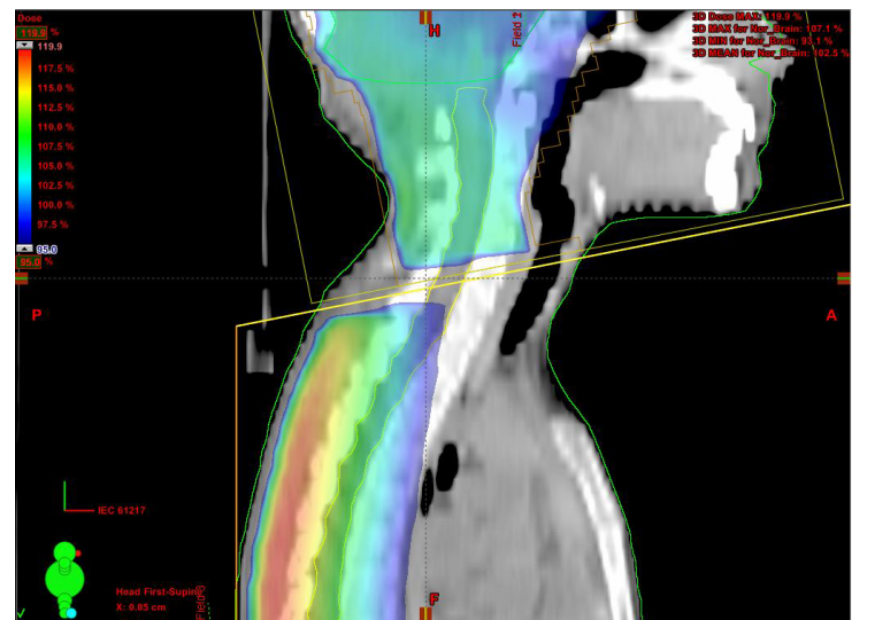

Figure 2. Simulation of matching junction technique demonstrating $2 \mathrm{~mm}$ gap leads to $30 \%$ underdose at the junction. 


\section{Treatment Planning and Delivery}

The planning objective was to deliver $36 \mathrm{~Gy}$ in 20 fractions to the PTV_Brain and PTV_Spine, to be followed by 18 Gy in 10 fractions to the PTV_Boost.

In order to achieve this, two plans, designed to treat simultaneously, were created with different isocentres. The isocentre of the first plan (Plan1_Day1) was identified around the $\mathrm{C} 2$ vertebra and the isocentre of the second plan (Plan2_Day1) was usually placed around the lower thoracic/upper lumbar vertebra region. Plan1_Day1 had a single isocentre and consisted of three beams - two lateral parallel-opposed skull fields and a posterior thorax field (using asymmetric jaws) - and covered the entire skull and the cervico-thoracic spinal regions. The second plan (Plan2_Day1) consisted of a single posterior beam which covered the remaining thoraco-lumbo-sacral region. The cranial beam edge of Plan2_Day1 matched the caudal beam edge of Plan1_Day1 at the level of the vertebral spine with a gap between the field edges, as defined by the field light on the skin.

In order to ensure appropriate coverage and to facilitate junction shifts, all the 3 fields of the Plan1_Day1 (having a single isocentre) were first normalized to the target mean of the PTV_Brain. Then, the weighting of the posterior cervicothoracic field of Plan1_Day1 was adjusted so as to obtain desired coverage of the PTV_Brain as well as of the cervicothoracic part of the PTV_Spine (95\% isodose line). In the other plan (Plan2_Day1) the dose to the single posterior beam was normalized such that the $95 \%$ isodose encompassed the thoraco-lumbo-sacral region. The coverage of target volumes for these set of plans was optimized using enhanced dynamic wedge and/or field-in-field methods, and irradiation of normal structures was reduced with the use of multi-leaf collimators (MLCs).

Both the plans were simply duplicated twice with no shift of either isocentre; i.e. Plan1_Day2, Plan2_Day2 and Plan 1_Day3, Plan2_Day3 in order to simulate the delivery of the 'moving junctions' on the machine in an alternating manner, having a 3-day cycle. In order to practice the moving junction ('feathering') technique, the junctions in the 3 alternating plans were separated with a planned (although individualised) shift of about $3 \mathrm{~cm}$ (at the level of the vertebral spine) using asymmetric jaws.

The Plan Sum tool was utilized to generate a composite plan of all the 3 sets of plans, and the desired coverage of the PTV_Spine (95\% isodose) was ensured without any unacceptable hot-spots. If unacceptable hot spots appeared, we increased the number of junctions. This was particularly helpful in cases where we wanted to focally boost the spinal cord in areas of gross disease.

The radiation delivery to the PTV_Boost (18 Gy in $10 \mathrm{Fr}$ ) was planned in the routine manner of planning focal RT to the brain using a different isocentre and 2-3 (or more) beams as required with normalization to either the target mean or at the isocentre to ensure coverage by the $95 \%$ isodose line.
During RT delivery, the fields were shifted in the following manner: On the first day of RT delivery, Plan1_Day1 treated the skull and cervico-thoracic regions, with its caudal beam edge being fixed by asymmetric jaws. The cranial edge of the Plan2_Day1 overlapped the caudal edge of Plan1_Day1 (using asymmetric jaws) while its caudal beam edge was fixed to cover the remaining thoraco-abdomino-sacral region, thus keeping both the isocentres fixed in their respective positions. In order to treat the Plan2_Day1 posterior field, the couch is moved cranially after delivery of Plan1_Day1 by the distance between the two isocentres (as ascertained on the TPS).

On the second day of RT delivery using Plan1_Day2, only the caudal edge of posterior cervico-thoracic field of 'shifts' cranially by $3 \mathrm{~cm}$ compared to Plan1_Day1 keeping the same isocentre and keeping its cranial edge as well as the bilateral skull fields unchanged. Simultaneously, the cranial edge of the thoraco-abdomino-sacral field (i.e. Plan2_Day2) shifts cranially by the same $3 \mathrm{~cm}$ keeping its caudal edge constant. To deliver the posterior fields (i.e. Plan1_Day2 and Plan2_Day2) the couch moves cranially in the same way as on Day 1 of RT.

On the third day of RT delivery, the caudal edge of the superior spinal field and the cranial edge of the inferior spinal field (Plan1_Day3 and Plan2_Day3) shift cranially by another $3 \mathrm{~cm}$, retaining the same isocentres. Similarly, the couch movements replicate those on the previous 2 days.

On the fourth day of RT delivery, the fields come back to the situation of Day 1, and the cycle is repeated with a 3-day cycling time. Thus, none of the isocentres shift during the entire course of the treatment.

Figures 3 and 4 show the beam arrangements and dose distributions for a typical plan.

We created two set-up fields $\left(20 \times 20 \mathrm{~cm}^{2}\right.$ field size), around both the isocentres, at gantry angles of $0^{\circ}$ and $270^{\circ}$, to verify the patient alignment and isocentre position.

All the plans were scheduled in an electronic chart on the Record and Verification System (Figure 5).

After the delivery of 36 Gy in 20 fractions of the CSI, the boost plan (18 Gy in 10 fractions) was started without any interruption as per scheduled in the RT chart.

Extended dose rate films (EDR2) were used to simulate the treatment plans with moving junction's accuracy in the junctions (Figure 6). The EDR films were sandwiched in between the perspex slabs to simulate the patient and all the three days plans were exposed to the films. The films were developed using an automatic film processor for evaluation. During the evaluation, we found that the junctions were matched as per our treatment plans and validated this technique is safely deliverable to the patients. 


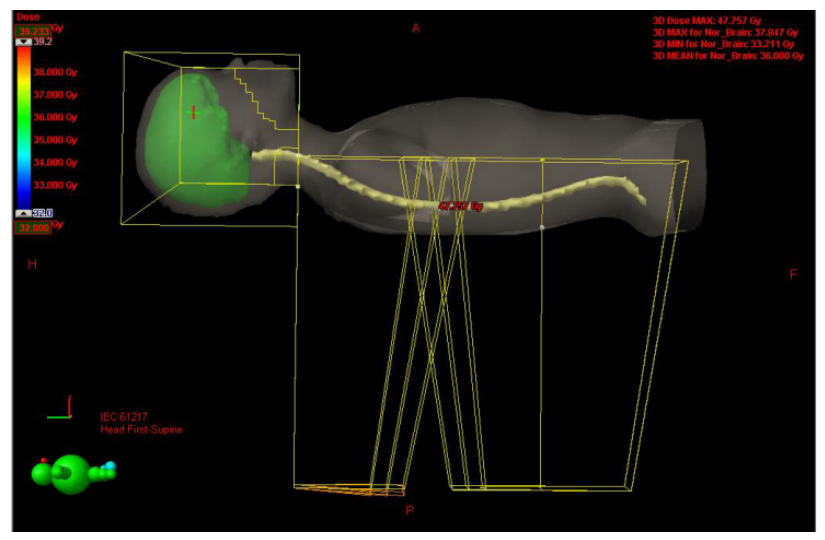

Figure 3. 3-Dimensional model view of beam arrangement.

\begin{tabular}{|c|c|c|c|c|c|c|c|}
\hline & Plan1_Day1 & |Plan1_Day2 & Plan1_Day3 & |Plan2_Day1 & Plan2_Day2 & |Plan2_Day3 & Boost \\
\hline 1 & Active & & & Active & & & \\
\hline 2 & & Active & & & Active & & \\
\hline 3 & & & Active & & & Active & \\
\hline 4 & Active & & & Active & & & \\
\hline 5 & & Active & & & Active & & \\
\hline 6 & & & Active & & & Active & \\
\hline 7 & Active & & & Active & & & \\
\hline 8 & & Active & & & Active & & \\
\hline 9 & & & Active & & & Active & \\
\hline 10 & Active & & & Active & & & \\
\hline 11 & & Active & & & Active & & \\
\hline 12 & & & Active & & & Active & \\
\hline 13 & Active & & & Active & & & \\
\hline 14 & & Active & & & Active & & \\
\hline 15 & & & Active & & & Active & \\
\hline 16 & Active & & & Active & & & \\
\hline 17 & & Active & & & Active & & \\
\hline 18 & & & Active & & & Active & \\
\hline 19 & Active & & & Active & & & \\
\hline 20 & & Active & & & Active & & \\
\hline 21 & & & & & & & Active \\
\hline 22 & & & & & & & Active \\
\hline 23 & & & & & & & Active \\
\hline 24 & & & & & & & Active \\
\hline 25 & & & & & & & Active \\
\hline 26 & & & & & & & Active \\
\hline 27 & & & & & & & Active \\
\hline 28 & & & & & & & Active \\
\hline 29 & & & & & & & Active \\
\hline 30 & & & & & & & Active \\
\hline
\end{tabular}

Figure 5. Plan scheduling in the radiotherapy treatment chart.

\section{Results and Discussion}

In this study, out of fifteen patients nine were male and 6 were female with a median age of 13.4 years (range 5-27 years). Treatment with anesthesia was given only to two patients. We found that only two patients failed in the primary site, no radiation myelitis or recurrences in the filed junctions were reported in these fifteen patients with a median follow-up of 36.4 months.

We demonstrated a simple supine cranio-spinal irradiation technique with inter fraction moving field junctions. This method is simple and practically deliverable with less chance of delivery error, and causes less discomfort to the patient than the prone method. Moreover, since the positioning is more stable, robust and reproducible, dosimetrically it appears to be more reliable than the pure field matching techniques which may potentially result in over or under-dosage.

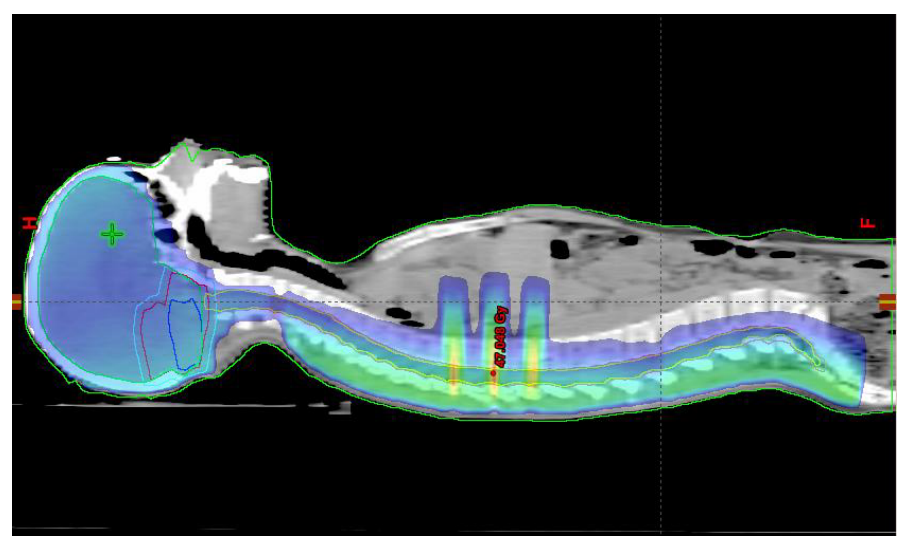

Figure 4. Sagittal multi-planar reconstruction of a patient with dose distribution.
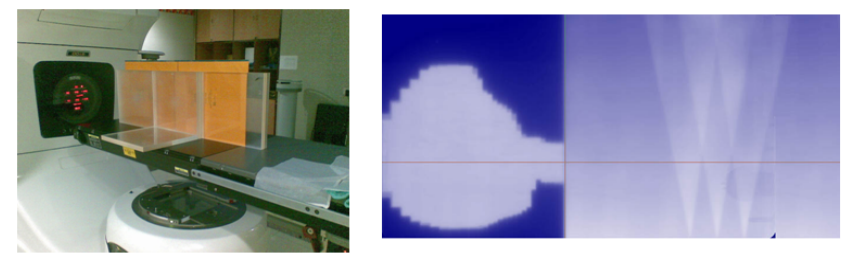

Figure 6. Treatment junction verification using EDR-2 films.

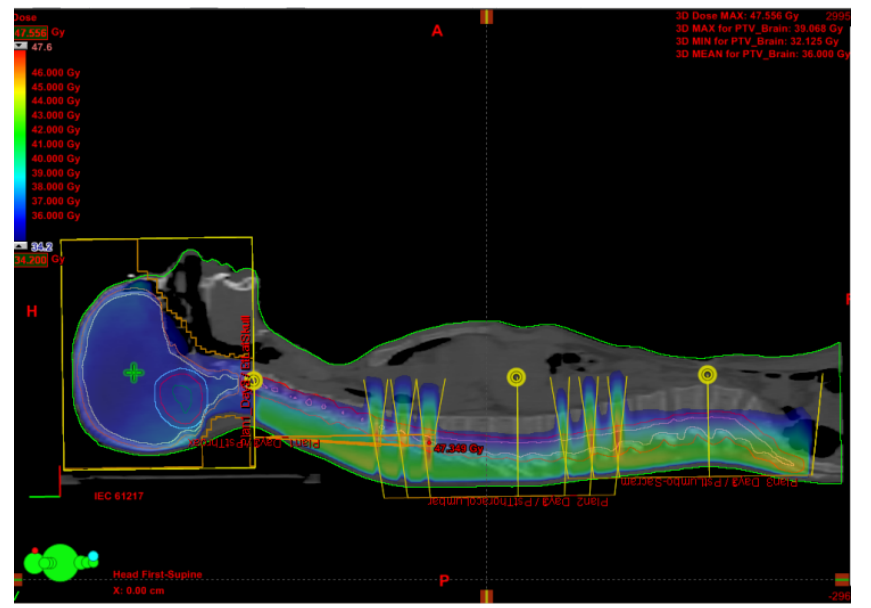

Figure 7. Beam arrangement and dose distribution of moving field junction technique in adults demonstrating 3 isocentres for extended spinal length coverage.

This technique is superior to the erstwhile moving junction technique since it employs a fixed set of isocentres throughout the course of delivery and is executed as per a pre-scheduled set of plans through a record and verify system, thereby neutralizing any element of technologist related delivery errors. Due to the combination of comfortable and reproducible supine positioning, use of CT data for dose calculation and planning, as well as availability of unique tools in various treatment planning systems for individualizing the dose distributions and coverage (and avoiding hot-spots), this technique ensures that the target volumes actually get adequate doses while avoiding possibly disastrous hot-spots.

This technique appears to be appropriate in children and short teenagers who constitute a vast majority of CSI patients, whereas in adults the adequate coverage can be ensured by simply increasing the number of moving junctions using an 
additional set of plans (e.g. Plan3_Day1, Plan3_Day2 and Plan3_Day3), with an additional isocentre, which will also remain constant throughout the treatment (Figure 7), such that the resultant posterior field adequately covers the remaining part of PTV_Spine. Care should be taken to ensure that the nomenclature of the plans and fields is clear and unambiguous and that sequencing and scheduling of the plans is entered accurately in the RT scheduling chart.

\section{Conclusion}

This supine technique for cranio-spinal irradiation demonstrated here is comfortable for the patient compared to the erstwhile prone position and also facilitates the delivery of anaesthesia where required for rigorous immobilization in treated children; it is easy to implement, saves time required for planning and treatment delivery and avoids possibility of treatment errors since all the isocentres are fixed throughout the course. Our cranio-spinal technique also comparable with Sue S. Yom et al [6] technique using intra-fraction modulated junction shifts where else we used inter-fraction junction shifts to improve the dose homogeneity and consistency of dose delivery, simplified treatment and reduces the impact of setup errors. Using 3D CT-based planning on advanced treatment planning systems accurate and safe dose delivery can be ensured as compared to the older conventional or the pure field-matching techniques.

\section{References}

[1] Halperin E. Impact of radiation technique upon the outcome of treatment for medulloblastoma. Int J Radiat Oncol Biol Phys. 1196;36(1):233-239.

[2] Parker WA, Freeman CR. A simple technique for cranio-spinal radiotherapy in the supine position. Radiother Oncol. 2006;78(2):217-222.

[3] Peñagarícano J, Moros E, Corry P, et al. Pediatric craniospinal axis irradiation with helical tomotherapy: patient outcome and lack of acute pulmonary toxicity. Int J Radiat Oncol Biol Phys. 2009;75(4):1155-1161.

[4] Skowrońska-Gardas A, Chojnacka M, Morawska-Kaczyńska M, et al. Patterns of failure in children with medulloblastoma treated with 3D conformal radiotherapy. Radiother Oncol. 2007;84(1):26-33.

[5] Taylor RE. United Kingdom Children's Cancer Study Group (UKCCSG) Radiotherapy and Brain Tumour Groups: Medulloblastoma/PNET and Craniospinal Radiotherapy (CSRT): Report of a Workshop held in Leeds, 30 June 1999. Clin Oncol. 2001;13(1):58-64.

[6] Yom SS. Frija EK, Mahajan A, et al. Field-In-Field Technique With Intrafractionally Modulated Junction Shifts for Craniospinal Irradiation. Int J Radiat Oncol Biol Phys. 2007;69(4);1193-1198.

[7] South M, Chiu JK, The BS, et al. Supine Craniospinal Irradiation Using Intrafractional Junction Shifts and Field-in-Field Dose Shaping: Early Experience at Methodist Hospital. Int J Radiat Oncol Biol Phys. 2008;71(2):477-483. 